\section{Alterations of the Corpus Callosum as an MR Imaging- Based Hallmark of Motor Neuron Diseases}

In motor neuron diseases (MNDs) such as amyotrophic lateral sclerosis (ALS) and primary lateral sclerosis (PLS), the definition of disease-related MR imaging characteristics is still ongoing. MR imaging approaches based on conventional and advanced techniques are frequently reported, aiming at the visualization of distinct regional patterns of structural or functional damage. Riad et $\mathrm{al}^{1}$ presented MR imaging visualization of the corticospinal tract (CST) and the motor segment of the corpus callosum (CC) in 2 patients with PLS, including region of interest-based quantifications of $\mathrm{T} 2$ hyperintense signal-intensity alterations in fluid-attenuated inversion recovery images. The authors compared their observations of circumscribed volume loss and signal-intensity alterations within the posterior body of the CC with the revised topographic scheme by Hofer and Frahm ${ }^{2}$ and thus confirmed the assumption of motor fiber distribution within this CC area.

However, Riad et $\mathrm{al}^{1}$ do not integrate this finding into the context of several recent studies on the pattern of structural brain alterations associated with different MNDs. In a recent study, ${ }^{3} 69$ patients with rare MND, including cohorts of 25 patients with PLS and 24 patients with pure hereditary spastic paraparesis (HSP), which are both characterized by a clinically prominent involvement of the upper motor neurons (UMNs), were investigated by computer-based MR imaging approaches using diffusion tensor imaging (DTI). Besides the observation of extensive areas of decreased fractional anisotropy (FA) within the CST in both MND and nonmotor brain regions in HSP, distinct patterns of microstructural integrity deterioration were identified both in PLS within the posterior body of the CC (analogous to the results of Riad et $\mathrm{al}^{1}$ ) and in HSP within dorsal parts of the CC to an even larger extent. These findings of microstructural disruption of the white matter tracts within the CC in MND with the clinical predominance of UMN impairment were confirmed by application of tractwise FA statistics of the callosal fiber distributions. ${ }^{3}$ In addition, by application of tract-based spatial statistics (www.fmrib.ox.ac.uk/ fsl), Filippini et $\mathrm{ll}^{4}$ recently reported the observation of decreased FA within the major tracts of the CC in patients with ALS, which extended rostrally and bilaterally to the region of the primary motor cortices.
Damaged tract integrity within the CC was also observed in patients with ALS by other groups. Beyond microstructural pathology visualized by DTI, decreased volume of the CC in patients with HSP was also observed to be localized in motor segment III, on the basis of voxel-based morphometry of T1-weighted 3D images. ${ }^{5}$ With respect to these findings within the CC motor section in PLS and HSP, it seems likely that these patients represent one end of a continuum in MND due to the long disease duration. In ALS with its much shorter survival times, microstructural damage (by DTI) but usually no macrostructural atrophy can be visualized.

Thus, advanced MR imaging analyses at a group level seem to be useful for the identification and quantification of pathoanatomic features in terms of noninvasive markers in MND, and DTI-based methods may reflect an additional tool for redefining the pathoanatomy of MND subtypes by microstructural in vivo fingerprint characteristics. In particular, the CC seems to be a key structure in the regional degeneration pattern in MND with a predominance of UMN affectation.

\section{References}

1. Riad SM, Hathout H, Huang JC. High T2 signal in primary lateral sclerosis supports the topographic distribution of fibers in the corpus callosum: assessing disease in the primary motor segment. AJNR Am J Neuroradiol 2011; 32:E61-E64

2. Hofer S, Frahm J. Topography of the human corpus callosum revisited-comprehensive fiber tractography using diffusion tensor magnetic resonance imaging. Neuroimage 2006;32:989-94

3. Unrath A, Müller HP, Riecker A, et al. Whole brain-based analysis of regional white matter tract alterations in rare motor neuron diseases by diffusion tensor imaging. Hum Brain Mapp 2010;31:1727-40

4. Filippini N, Douaud G, Mackay CE, et al. Corpus callosum involvement is a consistent feature of amyotrophic lateral sclerosis. Neurology 2010;75: 1645-52

5. Kassubek J, Juengling FD, Baumgartner A, et al. Different regional brain volume loss in pure and complicated hereditary spastic paraparesis: a voxelbased morphometric study. Amyotroph Lateral Scler 2007;8:328-36

A. Unrath

A.C. Ludolph

J. Kassubek

Department of Neurology

University of Ulm

Ulm, Germany

DOI 10.3174/ajnr.A2500 